\title{
Focused Ion Beam Preparation Techniques for EFTEM Analysis
}

\author{
P. Gnauck, U. Zeile, G. Benner, A. Orchowski, W-D. Rau \\ LEO Electron Microscopy Group, Carl Zeiss S-M-T AG, D-73447 Oberkochen, Germany
}

We present application examples of site specific energy filtered TEM (EFTEM) analysis using advanced FIB specimen preparation techniques. Specifically, we address topics such as troughput and reliability enhancement by chemically assisted broad ion beam milling and on-line monitoring of the etch process. We discuss how integrated elemental analysis by EFTEM can be used to gain quantitative information on the broad variety of new material systems currently entering front end and back end of the IC manufacturing process line.

The accelerating pace of device integration results in extreme demands for quantitative analysis in process development, yield ramp-up and process control with spatial resolution and elemental sensitivity at the very limits of currently available instrumentation. Historically, the highest resolution performance of TEM analysis has been hampered by long turn around times for the required sample preparation. Meanwhile the routine use of focused ion beam (FIB) systems for "trench" and "lift-out" preparation techniques allows for an enormous increase in the efficiency of TEM analysis.

The standard FIB cross sectioning is basically a blind process. The sample surface is imaged with the FIB before cutting to determine the area of interest. Then the sample is milled and polished with predefined milling patterns. Without the possibility of simultaneous monitoring of the milling process the area of interest is easily destroyed. The capability of CrossBeam technology to image the sample in real time at high resolution during the ion milling process using a field emission e-beam enables the operator to perform extremely accurate site specific cross sections. The milling and polishing process can be stopped exactly at the detail of interest e.g. in the very center of a certain transistor in a semiconductor device. In TEM sample preparation the real time SEM imaging allows for very tight control of the sample thickness/transparency and the danger of destroying the fine lamella is reduced to a minimum [1]. The FIB lift-out technique allows thin membranes to be extracted from bulk material, which saves sample pre-thinning time and is very successful in the preparation of site specific cross sections and planar samples. To a large extent TEM sample preparation can be automated by using scripts and macros. However, the best compromise concerning time and accuracy is achieved if different samples are pre-thinned automatically overnight to a thickness of about $1 \mu \mathrm{m}$ and then polished manually under high resolution SEM observation.

For the final investigation of the TEM lamellae energy filtered analysis allows for optimum image contrast even for thicker samples or heavy material structures in back-end analysis since the chromatic blur from inelastically scattered electrons can be eliminated. By selecting appropriate energy loss windows element specific contrast enhancement and quantitative determination of 2D composition distributions can be achieved. Moreover, using the small probe size of current FEG instruments, spectroscopic analysis in STEM and nanoprobe modes can reveal composition and interface properties on the sub-nanometer scale.

References

[1] R. Langford, A. Petford-Long, P. Gnauck, Proc. M\&M 2002, Quebec Can., Vol. 8, Suppl. 2, 44 

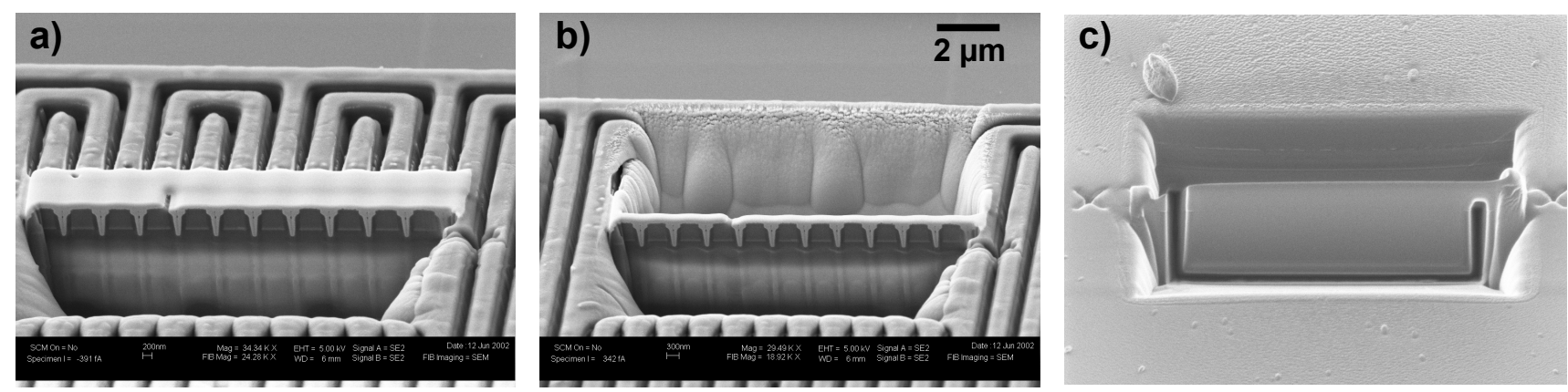

Fig. 1. Lift-out Procedure in Cross-Beam FIB Preparation: a) First side mill b) Second side mill and final polish c) After lift-out of the TEM lamella.

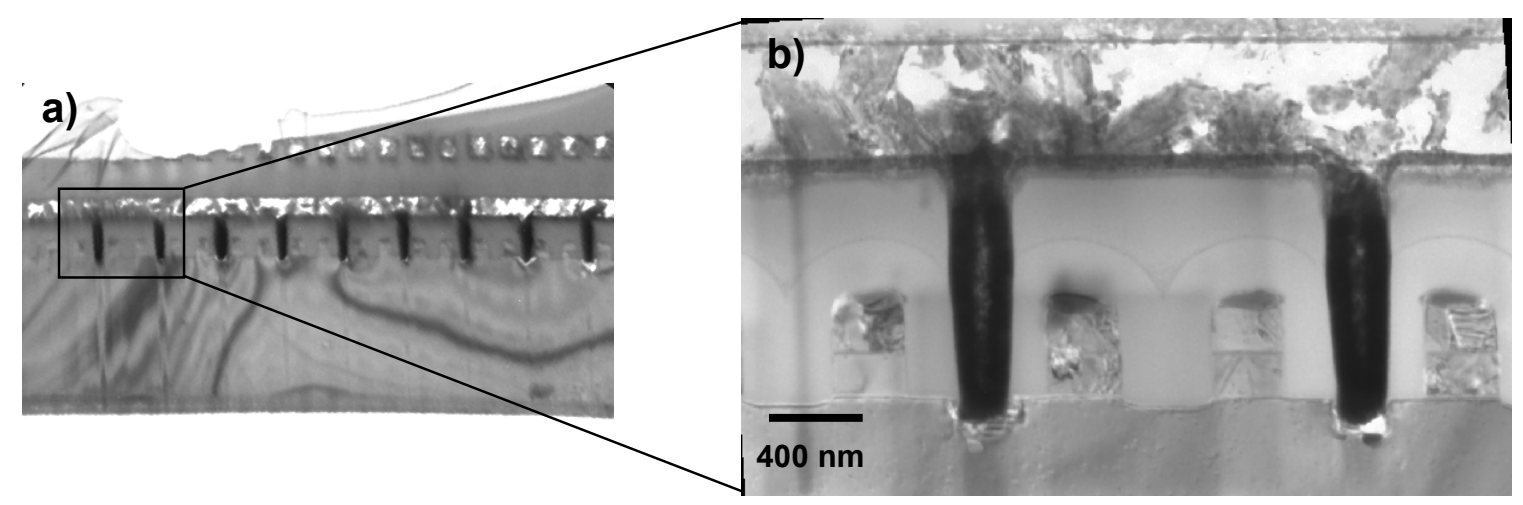

Fig. 2. TEM lamella of CMOS transistor contact plug region: a) Overview of lamella placed on carbon foil grid. b) Elastic image of detail area.
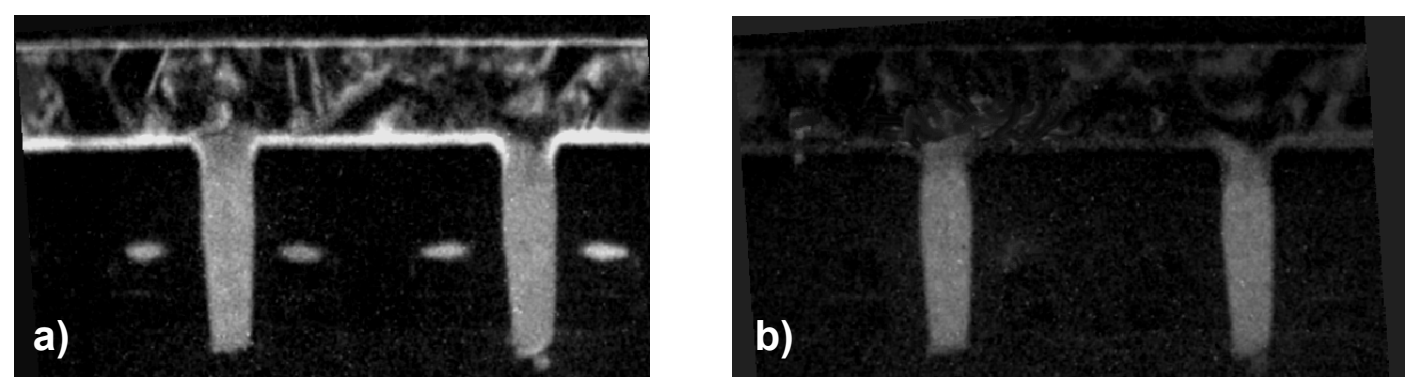

Fig. 3.Elemental distribution images of detail region in fig. 2: a) Titanium map b) Nitrogen map. 